\title{
Complete TURBT Before Radical Cystectomy Did Not Improve Oncological Outcome for Organ- Confined Bladder Cancer
}

Hai-tao Liang

Sun Yat-sen University Cancer Center

Zhan-ping Xu

Foshan hospital of TCM

Zhi-jun Lin

Sun Yat-sen University Cancer Center

Zi-ke Qin

Sun Yat-sen University Affiliated Tumor Hospital: Sun Yat-sen University Cancer Center

Yun-lin Ye ( $\nabla$ yeyunl@sysucc.org.cn )

Sun Yat-sen University Cancer Center https://orcid.org/0000-0001-5424-7066

Research

Keywords: complete TURBT, radical cystectomy, downstaging, bladder cancer.

Posted Date: December 23rd, 2020

DOI: https://doi.org/10.21203/rs.3.rs-131094/v1

License: (9) This work is licensed under a Creative Commons Attribution 4.0 International License. Read Full License 


\section{Abstract}

Background To investigate the role of complete transurethral resection of bladder tumor (TURBT) before radical cystectomy for organ-confined bladder cancer.

Patients and Methods Patients who underwent radical cystectomy (RC) in our center from January 2008 to December 2018 were retrospectively reviewed. Those with disease >T2NOM0 or positive surgical margin and those who were administrated neoadjuvant/adjuvant chemotherapy or radiotherapy were excluded. Complete TURBT was defined as no visible lesion under endoscopic examination after TURBT or specimen of RC. Kaplan-Meier and log-rank tests assessed disease-free survival. Logistic and Cox regression analysis were performed to identify potential predictors.

Results In total, 236 patients were included, and 207 patients were male. The median age was 61 years old. The median number and size were 1 and $3 \mathrm{~cm}$ respectively, and maximal pathological T stage was T2 in 94 patients. Complete TURBT was related to tumor size $(p=0.041)$, histological variants $(p=0.026)$ and downstaging $(p<0.001)$. Tumor size, grade and histological variants were independent predictors of complete TURBT. With a median follow-up of 42.7 months, 30 patients experienced recurrence. Age and histological variantswere independent predictors of disease-free survival $(p=0.022$ and 0.032 , respectively), and complete TURBT was not an independent predictor of disease-free survival $(p=0.156)$. Downstaging was not associated with survival outcome.

Conclusions Complete TURBT is related to an increased rate of downstaging before radical cystectomy, and it was not associated with better oncological outcomes for patients with organ-confined bladder cancer.

\section{Background}

Transurethral resection of bladder tumor (TURBT) is often the initial treatment for bladder cancer. Given its role in treatment and diagnosis, the extent and depth of TURBT, as well as the pathological result are important for management via intravesical instillation, neoadjuvant therapy and radical cystectomy [13]. Radical cystectomy (RC) is the standard option for muscle invasive bladder cancer (MIBC) and some high-risk non-muscle invasive bladder cancer (NMIBC). For locally-advanced disease, neoadjuvant chemotherapy is recommended, and downstaging is associated with good prognosis [4-9]. For $\leq$ PT2NOMO disease, complete TURBT was related to a higher rate of downstaging after RC, and it was associated with good outcomes [10-13]. However, in a recent study, the role of complete TURBT in oncological outcome was not significant, and incomplete TURBT was associated with a higher rate of pT3/4, which was a greater predictor of worse outcome than incomplete TURBT $[14,15]$. In clinical practice, advanced stage tied to poor prognosis, as well as incomplete TURBT. In contrast to pT3-4 bladder cancer, $\leq \mathrm{pT} 2 \mathrm{~N} 0 \mathrm{M} 0$ disease could be potentially completely dissected through TURBT. However, whether these patients could benefit from complete TURBT before RC is controversial. 
Hence, we evaluated the role of complete TURBT before radical cystectomy in patients with maximal pathological stage $\leq$ T2NOMO bladder cancer, in which radical TURBT was possible. Given that its role was most important for counseling prior radical cystectomy, TURBT parameters and the final pathological result after RC were taken into analysis. In addition, >T2NOMO disease was not included in this study, because complete resection was not always achieved using TURBT in these patients. To avoid related biases, patients with neoadjuvant chemotherapy/radiotherapy were excluded, and the duration between last TURBT and RC was less than 6 weeks.

\section{Methods}

Patients with bladder cancer treated in our cancer center from January 2008 to December 2018 were retrospectively reviewed. Of them, 526 cases underwent $\mathrm{RC}$ and urinary diversion in 4 weeks after last TURBT. In total, 287 cases were excluded for stage > T2NOM0, positive surgical margin, or neoadjuvant chemotherapy or radiotherapy. Three patients died in 30 days after RC were excluded. Clinical characteristics, imaging, pathology, and follow-up were retrospectively collected from our bladder cancer database.

Complete TURBT was defined as: no visible lesion under endoscope examination after TURBT, and no gross tumor in the bladder specimen after RC. Downstaging was defined as a T stage after TURBT that was greater than the stage after RC, and upstaging was defined as a T stage after RC that was greater than the stage after TURBT.

Disease-free survival was the primary endpoint. Continuous and categorical variables were presented as medians (interquartile range; IQR) and numbers (\%), and the comparisons between these variables were performed using the Mann-Whitney $\mathrm{U}$ test and Chi-square test. Kaplan-Meier and log-rank tests were calculated for survival analysis. Multivariate logistic and Cox regression analyses were performed for all variables that were identified as potentially significant by univariate analysis. Data were analyzed using IBM-SPSS Statistics ${ }^{\circledR}$, version 24 (IBM-Corp., Armonk, NY). All tests were two-tailed; $p<0.05$ was considered significant.

\section{Results}

In total, 236 patients were included, and 207 were male. The median age was 61 (53-69) years old. Median tumor number and size were $1(1-2)$ and $3(2-5) \mathrm{cm}$, respectively, and the pathological T stage was T2 in 94 patients. Complete TURBT and downstaging were accomplished in 143 and 25 cases, respectively. The downstaging rate was $17 \%$ in complete TURBT group, and $1 \%$ in the incomplete TURBT group ( $p<0.001$ ) (Table 1). And no adjuvant chemotherapy or radiotherapy was administrated for these organ-confined bladder cancer after radical cystectomy.

With Chi-square test, complete TURBT was related to tumor size $(p=0.041)$, variants $(p=0.026)$, downstaging $(p<0.001)$ and upstaging $(p<0.001)$, and high grade and LVI were marginally associated 
with complete TURBT (Table 1). Second TURBT was related to a higher rate of complete TURBT (69\% vs. $58 \%, p=0.149$ ), and this result was not significant. Second TURBT was significantly related to downstaging (14/184 vs. $11 / 52, p=0.005)$ (Table $1 S)$.

With a median follow-up of 42.7 (29.4-85.7) months, $13 \%$ (30/236) patients suffered relapse, the duration between RC and recurrence was from 3.2-131.3 months, with a median time of 22.2 months. Most (28/30) recurrence was detected 4 years after RC, and 16 tumors recurred in 2 years.

Based on Table 1, logistic analysis revealed that tumor size, grade and histological variants were independent predictors of complete TURBT (Table 2S). In univariate analysis, age, histological variants, T stage and complete TURBT were potentially associated with disease-free survival (DFS) $(p<0.13)$. In multivariate analysis, independent predictors of DFS were age, variants $(p=0.022$ and 0.032 , respectively), and complete TURBT was not an independent predictor of disease-free survival $(p=0.156)$ (Table 2, Fig. 1). 
Table 2

Univariate and multivariate analysis of potential factors for disease-free survival in organ-confined bladder cancer

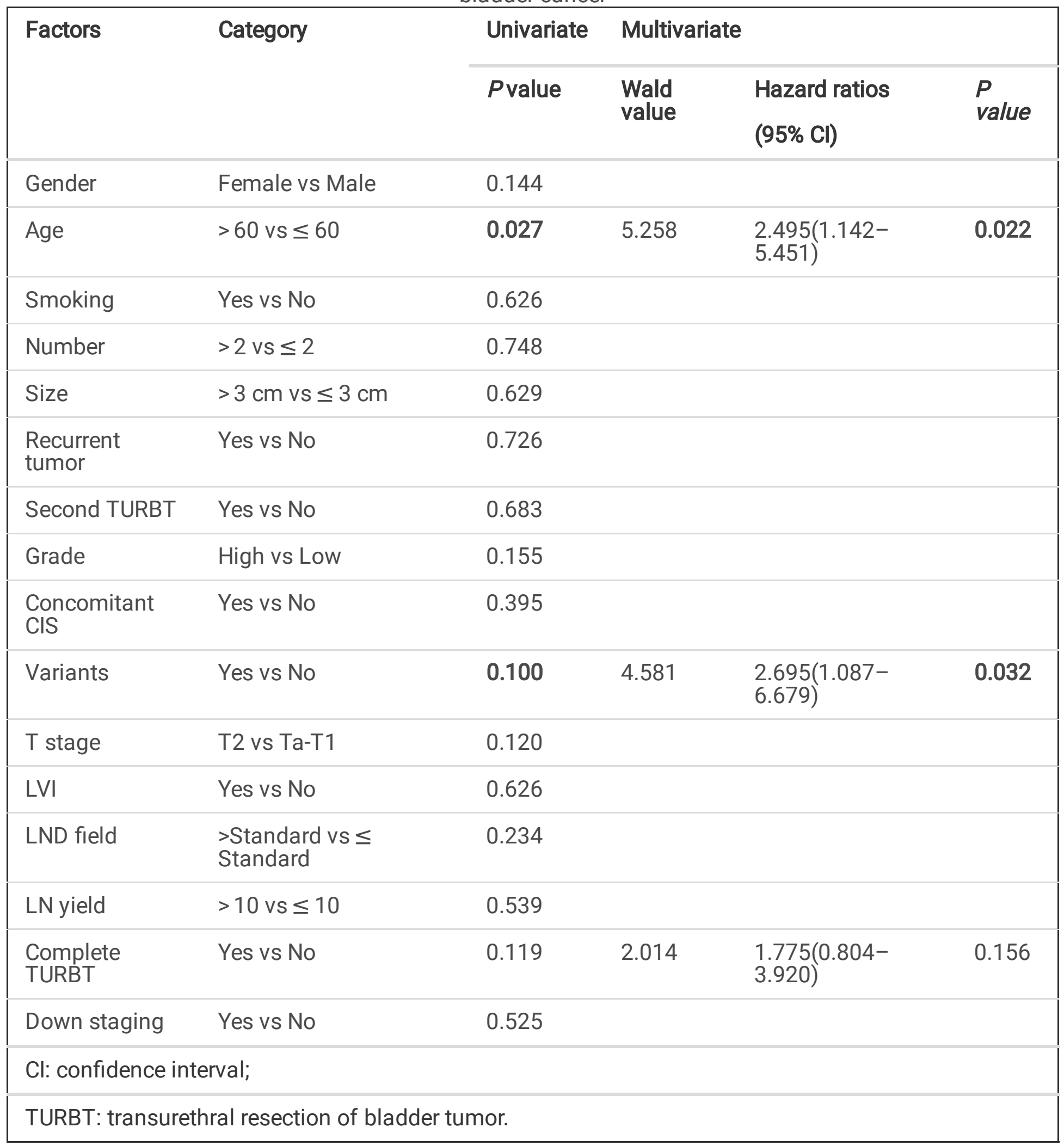

\section{Discussion}


The role of complete TURBT for NMIBC before intravesical therapy and repeat TURBT are confirmed by several studies $[1-3,16,17]$. However, its role before radical cystectomy $(R C)$ is controversial. And the completeness of TURBT was affect by tumor burden, which was also associated with oncological outcome $[9,10,14]$. In several retrospective analyses, complete TURBT was associated with a higher rate of downstaging, which was related to good prognosis $[10,11]$. For locally-advanced tumors, complete TURBT was not theoretically possible, and incomplete TURBT was often related to advanced stage (T34), which was a predictor of poor prognosis [14]. Thus, whether advanced stage or incomplete TURBT is a more effective predictor of prognosis was assessed. A recent study revealed that advanced stage was a greater predictor than incomplete TURBT, but some biases was inevitable, such as adjuvant therapy, positive surgical margin, and relatively subjective definition of complete TURBT [14]. Thus, we focused on organ-confined bladder cancer (Ta-T2), for which complete TURBT was available, to investigate the role of complete TURBT for RC candidates with organ-confined bladder cancer. For these patients, the primary tumor could be removed completely with RC, the role of complete TURBT would guide clinical practice for RC candidates. If complete TURBT did not improve outcome for these patients, it should be performed with caution to avoid related morbidities. In contrast, complete TURBT should be accomplished as much as possible before RC to improve oncological outcome.

In this study, complete TURBT was related to histological variants, tumor size, downstaging and upstaging. Using logistic analysis, we found that grade, size and histological variants were independent predictors of complete TURBT. These findings demonstrated that the increased tumor size and variant histology were associated with reduced rates of complete TURBT. Second TURBT was not associated with complete TURBT, but was associated with downstaging. Survival analysis results demonstrated that age, complete TURBT and histological variants were independent predictor of disease-free survival (DFS), and downstaging was not associated with lower rates of recurrence. Our study revealed that RC candidates with organ-confined bladder cancer did not benefit from complete TURBT(Fig. 1).

In studies of neoadjuvant chemotherapy for locally-advanced bladder cancer, downstaging and complete response were associated with good outcome. For those patients yielding downstaging, neoadjuvant chemotherapy was related to a better outcome than TURBT alone [5, 9]. These studies revealed that the response to chemotherapy and TURBT before RC toed to better cancer control. But the role of complete TURBT was not significant in some cohorts in which early-stage disease was more effective predictor of prognosis than complete TURBT [14]. In addition to tumor stage, adjuvant therapy and surgical margin status also affected outcome. In this study, these factors were excluded, and we found that complete TURBT was associated with worse DFS, as well as age and T stage, though it was not statistically significant.

Theoretically, complete TURBT might reduce the possibility of field seeding by decreasing tumor exposure during the operation. Additionally, complete TURBT was associated with early-stage disease and less tumor burden, so the role of complete TURBT in oncological outcome was covered. As reported, the circulating cancer cells increased during TURBT, and complete TURBT exhibited potential to increase tumor cell spreading, especially when bladder perforation occurred [18]. Compared to TURBT, RC could 
remove bladder cancer radically for organ-confined disease and is considerably more reliable than complete TURBT $[15,19]$. Based on this study, we had confirmed that complete TURBT did not improve survival for organ-confined bladder cancer patients before RC. And the bias between two groups, such as higher proportion of LVI, concomitant CIS and high-grade tumor, were substantially presented.

In most studies, downstaging had an important impact on survival, even neoadjuvant chemotherapy was not performed $[4,20,6]$. In this study, complete TURBT was related to a higher rate of downstaging, which was from $\mathrm{T} 2 / \mathrm{T} 1$ to $\mathrm{T} 1 / \mathrm{Ta}$, and downstaging was not significantly associated with oncological outcome significantly. In Hautmann's research, a series of RC was performed without neoadjuvant chemotherapy, and patients with maximal tumor stage pT2aNO had worse survival than patients with preoperative T2aNO and downstaging by TURBT [20]. However, multivariable analysis was not available in this large population study to confirm the role of downstaging by TURBT, and tumor stage and size were associated with both downstaging and survival. In this study, it was possible that all tumors could be resected completely by $\mathrm{RC}$ regardless of downstaging, so the role of complete TURBT and downstaging was not significant to oncologic outcome.

Some advantages of this study included the fact that biases of neoadjuvant/adjuvant therapy, tumor stage and positive surgical margin were excluded, and a relative long-term follow-up was available in this cohort. In addition, the limitations of this retrospective analysis should be mentioned. All cases received care in a single cancer center, and TURBT and RC were performed by different surgeons, who were primarily residents, but the quality was supervised by experienced surgeons. And second TURBT was not performed under consistent criteria. What's more, these data were prospectively collected from our Bladder Cancer database and follow-up was regularly updated. Finally, all recurrences were collected in this study, and local recurrence alone is rare. However, our study provided reliable information to evaluate the role of complete TURBT for RC candidates with organ-confined disease.

\section{Conclusions}

Complete TURBT is related to a higher rate of downstaging before radical cystectomy, and don't improve oncological outcomes for patients with organ-confined bladder cancer. For cystectomy candidates, radical TURBT might not be necessary.

\section{List Of Abbreviations}

TURBT: transurethral resection of bladder tumor

$\mathrm{RC}$ : radical cystectomy

NMIBC: non-muscle invasive bladder cancer

MIBC: muscle invasive bladder cancer 
IQR: inter-quartile range

DFS: disease-free survival

\section{Declarations}

\section{Ethics approval and consent to participate}

All procedures performed in studies involving human participants were in accordance with the ethical standards of the institutional and with the 1964 Helsinki declaration and its later amendments or comparable ethical standards. Writing informed consent from the patients and approval from the Sun Yat-sen University Cancer Center Institute Research Ethics Committee (approval number: GZR2018-053) was obtained.

\section{Consent to publish}

Not applicable.

\section{Availability of data and materials}

The authenticity of this article has been validated by uploading the key raw data onto the Research Data Deposit public platform (www.researchdata.org.cn), with the approval RDD number as RDDA2020001563.

\section{Competing interests}

The authors declare that they have no conflict of interests.

\section{Funding}

This study was supported by the Fundamental Research Funds for the Central Universities (Grant NO. 20ykpy179) and Medical Science Funding of Guangdong province (A2020139).

\section{Authors' contributions}

HTL, ZFC, ZPX and YLY were responsible for data collection and analysis, interpretation of the results, and writing the manuscript. YLY and ZKQ were responsible for conducting the study design, data analysis and interpretation. All authors have read and approved the final manuscript.

\section{Acknowledgments}

Not applicable.

\section{References}


1. Babjuk M, Burger M, Comperat EM, et al. European Association of Urology Guidelines on Non-muscleinvasive Bladder Cancer (TaT1 and Carcinoma In Situ) - 2019 Update. Eur Urol. 2019;76:639-657.

2. Flaig TW, Spiess PE, Agarwal N, et al. Bladder Cancer, Version 3.2020, NCCN Clinical Practice Guidelines in Oncology. J Natl Compr Canc Netw. 2020;18:329-354.

3. Richterstetter M, Wullich B, Amann K, et al. The value of extended transurethral resection of bladder tumour (TURBT) in the treatment of bladder cancer. BJU Int. 2012;110:E76-79.

4. Martini A, Jia R, Ferket BS, et al. Tumor downstaging as an intermediate endpoint to assess the activity of neoadjuvant systemic therapy in patients with muscle-invasive bladder cancer. Cancer. 2019;125:3155-3163.

5. Grossman HB, Natale RB, Tangen CM, et al. Neoadjuvant chemotherapy plus cystectomy compared with cystectomy alone for locally advanced bladder cancer. N Engl J Med. 2003;349:859-866.

6. Peyton CC, Tang D, Reich RR, et al. Downstaging and Survival Outcomes Associated With Neoadjuvant Chemotherapy Regimens Among Patients Treated With Cystectomy for Muscle-Invasive Bladder Cancer. JAMA Oncol. 2018;4:1535-1542.

7. Rosenblatt R, Sherif A, Rintala $E$, et al. Pathologic downstaging is a surrogate marker for efficacy and increased survival following neoadjuvant chemotherapy and radical cystectomy for muscle-invasive urothelial bladder cancer. Eur Urol. 2012;61:1229-1238.

8. Pignot G, Houede N, Roumiguie M, et al. [ypTONO after neoadjuvant chemotherapy and cystectomy for muscle-invasive bladder cancer: Incidence and prognosis. A review from the Bladder group of the French Committee of Oncology]. Prog Urol. 2018;28:567-574.

9. Cajipe $\mathrm{M}$, Wang $\mathrm{H}$, Elshabrawy $\mathrm{A}$, et al. Pathological downstaging following radical cystectomy for muscle-invasive bladder cancer: Survival outcomes in the setting of neoadjuvant chemotherapy versus transurethral resection only. Urol Oncol. 2020;38:231-239.

10. Lee SE, Jeong IG, Ku JH, Kwak C, Lee E, Jeong JS. Impact of transurethral resection of bladder tumor: analysis of cystectomy specimens to evaluate for residual tumor. Urology. 2004;63:873-877; discussion 877.

11. van Dijk PR, Ploeg M, Aben KK, et al. Downstaging of TURBT-Based Muscle-Invasive Bladder Cancer by Radical Cystectomy Predicts Better Survival. ISRN Urol. 2011;2011:458930.

12. Nielsen ME, Bastian PJ, Palapattu GS, et al. Recurrence-free survival after radical cystectomy of patients downstaged by transurethral resection. Urology. 2007;70:1091-1095.

13. Volkmer BG, Kuefer R, Bartsch G Jr, et al. Effect of a pT0 cystectomy specimen without neoadjuvant therapy on survival. Cancer. 2005;104:2384-2391.

14. Zamboni S, Moschini M, Gallina A, et al. The impact of completeness of last transurethral resection of bladder tumors on the outcomes of radical cystectomy. World J Urol. 2019;37:2707-2714.

15. Kukreja JB, Porten S, Golla V, et al. Absence of Tumor on Repeat Transurethral Resection of Bladder Tumor Does Not Predict Final Pathologic T0 Stage in Bladder Cancer Treated with Radical Cystectomy. Eur Urol Focus. 2018;4:720-724. 
16. Divrik RT, Yildirim U, Zorlu F, Ozen $\mathrm{H}$. The effect of repeat transurethral resection on recurrence and progression rates in patients with $\mathrm{T} 1$ tumors of the bladder who received intravesical mitomycin: a prospective, randomized clinical trial. J Urol. 2006;175:1641-1644.

17. Grimm MO, Steinhoff C, Simon X, Spiegelhalder P, Ackermann R, Vogeli TA. Effect of routine repeat transurethral resection for superficial bladder cancer: a long-term observational study. $J$ Urol. 2003;170:433-437.

18. Engilbertsson $\mathrm{H}$, Aaltonen $\mathrm{KE}$, Bjornsson $\mathrm{S}$, et al. Transurethral bladder tumor resection can cause seeding of cancer cells into the bloodstream. J Urol. 2015;193:53-57.

19. Bus MT, Cordeiro ER, Anastasiadis A, Klioueva NM, de la Rosette JJ, de Reijke TM. Urothelial carcinoma in both adnexa following perforation during transurethral resection of a non-muscleinvasive bladder tumor: a case report and literature review. Expert Rev Anticancer Ther. 2012;12:1529-1536.

20. Hautmann RE, de Petriconi RC, Pfeiffer C, Volkmer BG. Radical cystectomy for urothelial carcinoma of the bladder without neoadjuvant or adjuvant therapy: Iong-term results in 1100 patients. Eur Urol. 2012;61:1039-1047.

\section{Tables}

Due to technical limitations, table 1 is only available as a download in the Supplemental Files section.

\section{Figures}
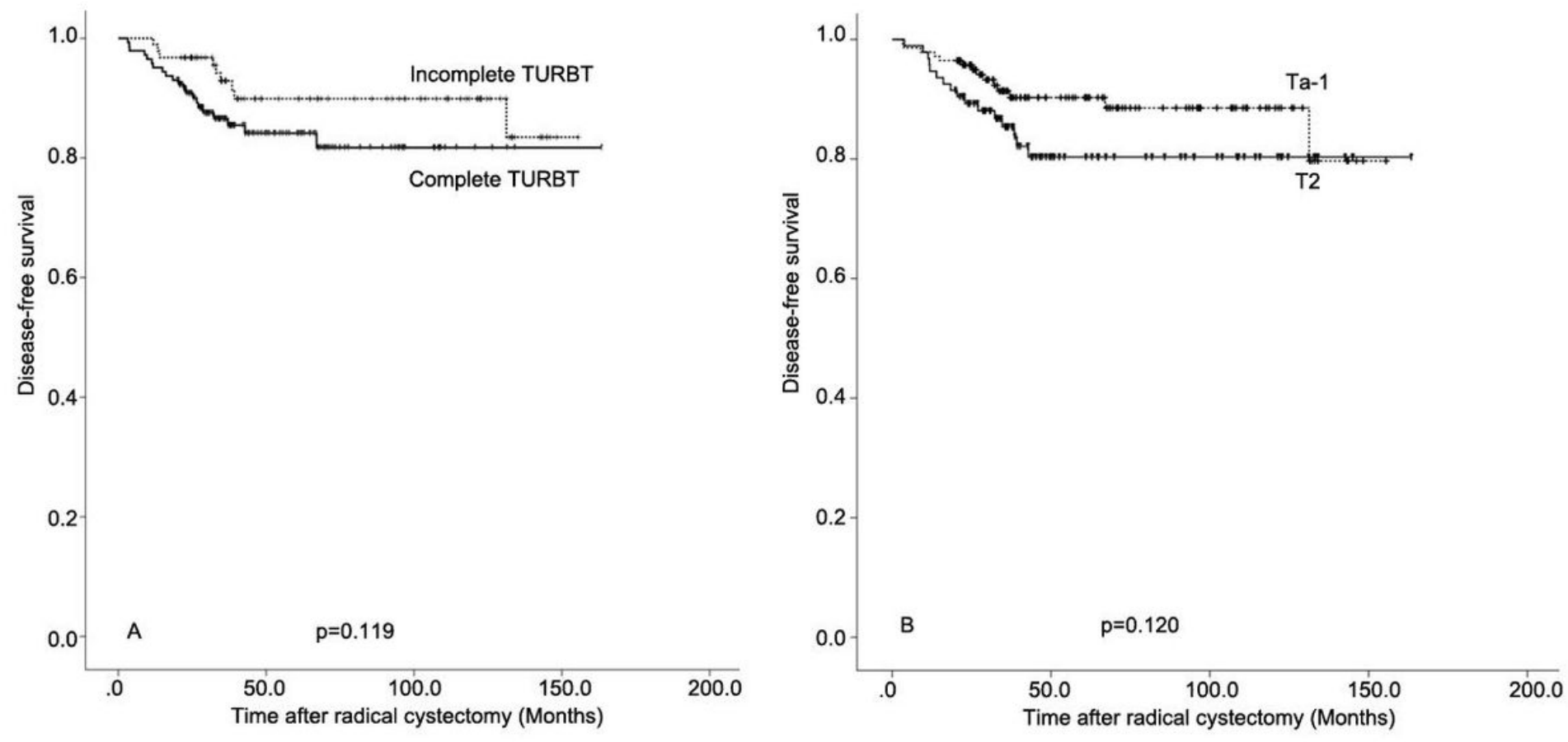

Figure 1 
Disease-free survival analysis of different subgroups A: Disease-free survival stratified by complete TURBT status; B: Disease-free survival stratified by maximal pathological T stage.

\section{Supplementary Files}

This is a list of supplementary files associated with this preprint. Click to download.

- Table1.pdf

- Table1Sdownstaging.pdf

- Table2ScTURpredictor.pdf 